\title{
The Jones-Witten Invariant for Flows on a 3-Dimensional Manifold
}

\author{
Alberto Verjovsky ${ }^{1}$, Ricardo F. Vila Freyer $^{2}$ \\ 1 International Centre for Theoretical Physics, P.O. Box 586, I-34100 Trieste, Italy \\ 2 CIMAT, Apdo. Postal 402, 36000 Guanajuato, Gto., México
}

Received: 6 October 1992/in revised form: 29 November 1993

\begin{abstract}
The Jones-Witten functional for knots is generalized for smooth flows on 3-dimensional manifolds. Explicit computations for the abelian case are given.
\end{abstract}

\section{Introduction}

Recently Witten has proposed a generalization of the Jones polynomial for links which has the advantage of being intrinsically defined on any closed orientable 3-manifold (see [W] and [At]). Witten defines his invariant via holomorphic sections of a vector bundle on a canonical moduli space related to a Heegard splitting of the 3-manifold. He develops this theory from the Hamiltonian point of view, and then he gives an interpretation of the Jones polynomial in terms of a 3-dimensional Yang-Mills theory, where the Lagrangian $\mathscr{L}$ is the functional which assigns to each connection its ChernSimons character, with weight $k$ (an integer or half integer for the $S U(N)$ or the $U(1)$ theories respectively):

$$
\begin{gathered}
\mathscr{L}: \mathscr{C} \rightarrow \mathbb{R} / \mathbb{Z}, \\
\mathscr{L}_{k}(A)=\frac{k}{8 \pi} \int_{M} \operatorname{tr}\left(A \wedge d A+\frac{2}{3} A \wedge A \wedge A\right),
\end{gathered}
$$

where $\mathscr{A}$ is the space of all connections on the trivial principal bundle $P=M \times G \rightarrow$ $M$, and the structure group is either $S U(N)$ or $U(1)$. The trace corresponds to the bilinear Killing form for the compact gauge group under some explicit representation of the group.

Our purpose in this note is to extend Witten's ideas to construct new topological invariants of a smooth dynamical system, namely invariants under differentiable equivalence. A possible physical interpretation of our formula case is as an averaged Bohm-Aharonov effect for a "continuous" flux of electrons. 
One might think of a non-singular flow together with flow-invariant measures as a generalization of a link, namely, as a "diffuse" link on the manifold. For instance, if a given flow contains periodic orbits $\Gamma_{1}, \Gamma_{2}, \ldots, \Gamma_{n}$; and if we consider the invariant probability measure $\mu=\mu_{1}+\mu_{2}+\ldots+\mu_{n}$; where the $\mu_{i}$ is supported on $\Gamma_{i}$, and uniformly distributed with respect to the time parameter on $\Gamma_{i}$, then our invariant essentially coincides with Witten's for the link whose components are the $\Gamma_{i}$ 's. One might think of these measures as Dirac measures supported along the periodic orbits. On the other hand, if the flow-invariant measure has as support the whole manifold $M$, then one can think of the flow as a "diffuse" link.

In order to define our invariant one must analyze the asymptotic behavior of an arc of trajectory as it wraps around the manifold and understand the evolution of the holonomy along this path (we think of the orbits of the flow as the Wilson lines), and study the average behaviour with respect to given invariant measures.

The central idea of assigning an asymptotic invariant originates with Schwartzman's beautiful paper [Sc] on asymptotic cycles in which he defines a real homology class corresponding to the average homological "placement" of the orbits of the flow. Later this theory was developed by Sullivan [Su] who interprets a flow and in general a foliation as a de Rham current and introduces the idea of foliated cycles. For instance an irrational flow on the torus can be both interpreted as a uniquely ergodic flow or as a "diffuse" cycle. In this way, one might think of real homology classes as geometric objects. Another example is furnished by a foliation with a transverse measure introduced by J. Plante, which can be thought of as the cohomology class which assigns to each cycle of the complementary dimension transverse to the foliation the integral of the transverse measure along this cycle. Finally, Arnold [A] uses similar ideas to define the average asymptotic linking number of a flow that leaves invariant a measure defined by a volume form on the 3-manifold $M$, and proves that when the flow is homologically trivial, this coincides with a natural quadratic form on the Lie algebra of the group of volume preserving diffeomorphisms of $M$ that are invariant under the adjoint action of the group on the algebra.

One of our main results in the abelian case is to equate the generalized JonesWitten invariant of the flow with certain exponential with weight $k$ of this asymptotic linking number times a constant depending on the Ray-Singer torsion. In this way we are able to explicitly compute the invariant in some cases for manifolds which

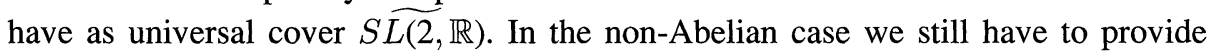
explicit computations and justify the meaning of the invariant. We leave this for a future paper.

The paper is organized as follows. In the first section we recall the Dynamical Systems and Ergodic theory required by introducing the Krylloff and Bogoliuboff results. In the second section we recall Schwartzman theory of Asymptotic Cycles and reinterpret it in terms of a $U(1)$ gauge theory for flat connections. Next we give Arnold's definition of the asymptotic Hopf invariant (the asymptotic average linking number of a flow). This provides the tool to show the interconnection as in Witten's case, of the regularization of the self-linking number of a flow and the choice of framing for the link on the 3-manifold. In the third section we recall Witten's definition of the Jones polynomial, partly formalized in the work of Ramadas, Singer, and Weitsman [RSW]. Then we define the invariant for the pair $(X, \mu)$ where $\mu$ is an invariant probability measure for the flow defined by the vector field $X$, and verify that this invariant is well defined as a limiting average of Witten's definition. We 
show that for the abelian case this is explicitly computable using Arnold's results. In the fourth section we give the definition of the invariant in the case the structure group is a simply connected non-abelian Lie group.

We are very grateful with E. Ghys who showed the first author how one can define the average genus of a flow in $S^{3}$, because this inspired the ideas of this paper. We are also grateful with L. Alvarez-Gaumé and J. Labastida for explaining to the second author the physicist's interpretation of the infinite dimensional gaussian integral in Witten's paper. We also would like to thank Prof. V. I. Arnold and M. Brunella for pointing out to us some references.

\section{Kryloff-Bogoliuboff Theory}

We review in this section some concepts necessary for our work. The main reference is $[\mathrm{Sc}]$ and the references therein.

Assume $M$ is a compact closed manifold, $X$ a smooth vector field on $M$ and $f_{t}$ the flow generated by it. We will denote by $\mathscr{B}(X)$ the set of Borel probability measures on $M$, invariant under translations by the flow (i.e. $\int_{M} g(x) d \mu(x)=\int_{M} g\left(f_{t}(x)\right) d \mu$, for any $g \in L^{1}(M, \mu), \mu \in \mathscr{B}(X)$ and $\left.t \in \mathbb{R}\right)$. It is known that $\mathscr{B}(X)$ is non-empty, convex, weak ${ }^{*}$-compact, where regular Borel signed measures are identified with $C(M)^{*}$ (the dual space of continuous functions on $M$ ) by the Riesz representation theorem.

Definition (Krylloff-Bogoliuboff). A point $p \in M$ is quasi-regular if the average value of any function under the flow through $p$ exists. Namely, if the integral:

$$
\lim _{T \rightarrow \infty} \frac{1}{T} \int_{0}^{T} g\left(f_{t}(p)\right) d t
$$

exists for any $g \in C(M)$. Then there exists a unique $f_{t}$-invariant probability measure $\mu_{p}$ such that this average equals:

$$
\int_{M} g(x) d \mu_{p}(x) .
$$

Furthermore, if we denote by $\mathscr{Q R}(X)$ the set of quasi-regular points of $M$ with respect to the flow generated by the vector field $X$, then it is invariant and of measure one for any measure in $\mathscr{B}(X)$.

A flow $f_{t}$ is ergodic with respect to a $f_{t}$-invariant measure $\mu$ if for any measurable set $\mathscr{W}$ in $M$, such that it remains invariant under the action of $f_{t}$ (i.e. if $f_{t}(\mathscr{W})=\mathscr{W}$ ), then either $\mu(\mathscr{\mathscr { C }})=1$ or $\mu(\mathscr{C})=0$. A flow is called uniquely ergodic if the $f_{t}$ invariant measure is unique, i.e. $\mathscr{B}(X)$ consists of one point.

1.1. Example. Let $T^{2}=\mathbb{R}^{2} / \Lambda$ be the torus generated by the translations $\alpha:(x, y) \rightarrow$ $(x+1, y)$ and $\beta:(x, y) \rightarrow(x, y+1)$, and $\Lambda=\langle\alpha, \beta\rangle$ be the lattice generated by $\alpha$ and $\beta$. Define the flow $f_{t}(x, y)=(x, y)+t(p, q)$. If $\lambda=p / q$ is a rational number, then the flow is periodic and its orbit through any point $x \in T^{2}$ will describe a knot winding around $p$-times in one direction and $q$-times in the other with respect to the canonical generators induced by $\alpha$ and $\beta \in H_{1}\left(T^{2}, \mathbb{Z}\right)$. In this case there are many 
invariant measures for which the flow is ergodic. For instance measures concentrated in any periodic orbit through a point.

If $\lambda$ is irrational, then it is known that the flow is minimal (i.e., every orbit is dense), and furthermore, that there exists a unique invariant measure (the standard Lebesgue measure) on $T^{2}$ for which $f_{t}$ is ergodic.

1.2. Example. If $\left.M^{3}=S \widetilde{L(2}, \mathbb{R}\right) / \Gamma$ is a 3 -manifold with $\Gamma$ a discrete uniform subgroup then it is known that the flows induced by the left-invariant vector fields of $\mathfrak{s l}(2, \mathbb{R}): X=\left(\begin{array}{ll}0 & 1 \\ 0 & 0\end{array}\right)$, and $Y=\left(\begin{array}{ll}0 & 0 \\ 1 & 0\end{array}\right)$ descend to uniquely ergodic flows in $M^{3}$ which are minimal (i.e. all its orbits are dense). If we denote the flows by $f_{t}$ and $g_{t}$, they are called the positive and negative horocyclic flows, respectively. The invariant measure is the volume generated by the duals of $X, Y$ and $H=\left(\begin{array}{cc}1 & 0 \\ 0 & -1\end{array}\right)$. The flow $h_{t}$ generated by $H$ is called the geodesic flow, it is ergodic (see [CFS]).

\section{Schwartzman asymptotic cycles (see [Sc, A, KC])}

2.1 We will consider a manifold $M$ together with a flow $f_{t}$ as in Sect. 1, a fixed set of regular curves $\left\{\gamma_{p, q}\right\}$ of uniformly bounded distance (say, less than 2 times the diameter of $M$ ) joining any two distinct points of $M$ (see Khesin and Chekanov [KC, Sect. 4]).

Through any point $p \in M$, and for any $t$ positive, we define the integral-singular 1-cycle represented by:

$$
\tilde{\Gamma}_{t, p}=\left[p, f_{t}(p)\right] \cup \gamma_{f_{t}(p), p}
$$

where $\left[p, f_{t}(p)\right]$ is the oriented arc of trajectory going from $p$ to $f_{t}(p)$.

Consider now the real 1-cycle $\Gamma_{t, p}=\frac{1}{t} \tilde{\Gamma}_{t, p}$. We represent its real homology class by $\left[\Gamma_{t, p}\right]$.

Theorem (Schwartzman). The limit $\lim _{t \rightarrow \infty}\left[\Gamma_{t, p}\right]=\left[\Gamma_{p}\right]$ exists in $H_{1}(M, \mathbb{R})$ for every quasi-regular point $p \in \mathscr{Q R}(X)$ and it is independent of the Riemannian metric of $M$ and the arbitrary choice of the connection curves $\gamma_{p, q}$.

Hence, we can think of $\left[\Gamma_{p}\right]$ as the "diffuse" link defined by the orbit of the flow $f_{t}$ through $p$. The homological contribution of the connecting curves $\gamma_{p, q}$ goes to zero by the uniform bound on their lengths and the division by $t$. Let $\Gamma: \mathcal{Q B}(X) \rightarrow H_{1}(M, \mathbb{R})$ be the function which sends $p$ to $\left[\Gamma_{p}\right]$. The cycle $\left[\Gamma_{p}\right]$ is called the asymptotic cycle through $p$ of the flow and $\Gamma$ the asymptotic cycle map. It gives an asymptotic behavior of the orbit of the flow through $p$ in terms of homology.

There is an alternative description of the asymptotic cycles given by Schwartzman in terms of the Eilenberg-Bruschlinsky homology theory that will enable us to translate into an abelian $U(1)$-gauge theory.

Let us identify $U(1)$ with $S^{1}=\{z \in \mathbb{C}:|z|=1\}$, and let $\mathscr{C}(M)=\mathscr{C}\left(M, S^{1}\right)$ denote the abelian group of continuous maps from $M$ to $S^{1}$ under pointwise multiplication, and $\mathscr{B}(M)$ denote the closed subgroup of $\mathscr{C}(M)$ consisting of

$$
\mathscr{B}(M)=\{f \in \mathscr{C}(M) \mid \exists h: M \rightarrow \mathbb{R} \text { such that } f(x)=\exp (2 \pi i h(x))\} .
$$

Theorem (Eilenberg-Bruschlinsky [Sc]). $\mathscr{C}(M) / \mathscr{B}(M)$ is algebraically isomorphic to $H_{1}(M, \mathbb{Z})$ in terms of $\check{C}$ ech cohomology. The isomorhism is given explicitly as 
follows. Let $g \in \mathscr{C}(M)$, and consider for any cycle (for instance $\tilde{\Gamma}_{t, p}$ as before) the restriction of $g$ to the cycle. This is a map from the cycle to $S^{1}$, and its degree is well defined. Then the pairing:

$$
\begin{gathered}
\mathscr{C}(M) / \mathscr{R}(M) \times H_{1}(M, \mathbb{Z}) \rightarrow \mathbb{Z}, \\
(g, C) \rightarrow \operatorname{deg}\left(\left.g\right|_{C}\right)
\end{gathered}
$$

is a duality pairing. If $C=\tilde{\Gamma}_{t, p}$, then the assignment:

$$
g \rightarrow \lim _{t \rightarrow \infty} \frac{1}{t}\left[\operatorname{deg}\left(\left.g\right|_{\tilde{\Gamma}_{t, p}}\right)\right]
$$

defines an element of $H_{1}(M, \mathbb{R})$ by duality.

Finally, as in Atiyah-Bott [AB], if we think of $\mathscr{C}(M)$ as the gauge group for the trivial principal bundle $P=M \times U(1)$, then the connections, because the bundle is trivial, can be thought of as a 1 -forms in $\Lambda^{1}(M) \otimes \mathfrak{u}(1)$. The kernel under exterior differentiation $d=d_{A}$ is the set of closed 1-forms with values in $\mathfrak{u}(1)$. Hence (Flat connections)/(Gauge transformations) is the same as $H^{1}(M, \mathbb{R}) / H^{1}(M, \mathbb{Z})$, i.e. the "moduli" of flat connections modulo the gauge group, and corresponds to the space of irreducible representations of $\pi_{1}(M) \rightarrow U(1)$. The space $\mathscr{C}(M) / \mathscr{B}(M)$, the Eilenberg-Bruschlinsky group, is the quotient of the Gauge group modulo the gauge transformations homotopic to the identity.

Asymptotic cycles can also be interpreted as winding cycles as follows. Let $\mu \in \mathscr{B}(X)$ and $w$ a closed 1-form representing a cohomology class $[w]$, in the de Rham cohomology. Define the functional

$$
\Psi_{\mu}: H_{D R}^{1}(M, \mathbb{R}) \rightarrow \mathbb{R}
$$

as follows:

$$
\Psi_{\mu}([w])=\int_{M} w(X) d \mu .
$$

Then $\Psi_{\mu}$ is well defined, it does not depend on the representing 1-form $w$, namely, if $\bar{w}=w+d f$; then $\Psi_{\mu}([\bar{w}])=\int_{M}(w(X)+d f(X)) d \mu=\Psi_{\mu}([w])+\int_{M} d f(X) d \mu$. And the last term is zero because the measure is invariant under translations by the flow. Then $\Psi_{\mu}$ is a linear functional in the de Rham cohomology and by duality defines a cycle, the winding cycle $\Psi_{\mu}$ in $H_{1}(M, \mathbb{R})$ for each $\mu \in \mathscr{B}(X)$. Therefore we have an affine map

$$
\Psi: \mathscr{B}(X) \rightarrow H_{1}(M, \mathbb{R})
$$

whose image is a compact convex subset of a finite dimensional vector space. When $X$ is uniquely ergodic $\mathscr{B}(X)$ reduces to one point $\mu$, and $\Psi$ determines a unique element $\Psi_{\mu}$ in $H_{1}(M, \mathbb{R})$ which we call the winding cycle.

2.2. Arnold [A] defines, using the idea of asymptotic cycle, the concept of asymptotic average linking number or asymptotic Hopf invariant of the flow.

In Arnold's case, $M$ is a closed orientable 3-manifold with invariant measure the volume form, $v=$ vol, which we will assume normalized so that $M$ has volume one, $X$ is a homologically trivial vector field and $v$ is invariant under the action of the flow $f_{t}$ induced by the vector field $X$. Invariance means that $\mathscr{L}_{X}(v)=d \cdot i_{X}(v)=0$. 
The homological triviality of $X$ means that there exists a 1 -form $\alpha$ such that if $\theta_{X}$ denotes the contraction $i_{X}(v)$ then $d \alpha=\theta_{X}$. Since

$$
\int_{M} w(X) \cdot v=\int_{M} w \wedge\left(i_{X}(v)\right)=\int_{M} w \wedge \theta_{X}=\int_{M} w \wedge d \alpha=-\int_{M} d(w \wedge \alpha)=0
$$

for any closed 1 -form $w$, one has that the winding cycle $\Psi_{v}$ is identically zero for a homologically trivial vector field with respect to an invariant volume form.

In this situation the linking number $L\left(\tilde{\Gamma}_{t, p}, \tilde{\Gamma}_{t, q}\right)$ exists and is well defined (and may depend on the closing curves $\gamma_{f_{t}(p), p}$ and $\gamma_{f_{t}(q), q}$ that we may choose to be disjoint for almost any pair of points $p$ and $q$ ). However, the asymptotic average linking number:

$$
\lim _{T_{1}, T_{2} \rightarrow \infty} \frac{1}{T_{1} \cdot T_{2}} L\left(\tilde{\Gamma}_{T_{1}, p}, \tilde{\Gamma}_{T_{2}, q}\right):=L\left(\Gamma_{p}, \Gamma_{q}\right)
$$

is well defined since the contribution of the linking of the joining curves can be kept bounded (via a good choice of joining curves $\gamma_{p, q}$ ) and becomes negligible with the limiting process, and is therefore independent on these curves. (For the details see Arnold's paper cited above, and also Sect. 4 on Khesin and Chekanov's paper.) So that we have:

Theorem (Arnold). The average asymptotic linking number or average Hopf invariant of the homologically trivial vector field $X$ on the compact closed 3-manifold $M$ :

$$
L(X)=\int_{M \times M} L\left(\Gamma_{p}, \Gamma_{q}\right) d v(p) d v(q)
$$

is well defined. Furthermore,

$$
L(X)=\int_{M} \alpha \wedge d \alpha
$$

where $\alpha$ is defined as above, namely, $d \alpha=i_{X}(v)$.

Generalizing from this result, Tabachnikov (in [T]) and Khesin and Chekanov (in $[\mathrm{KC}]$ ) define the asymptotic linking number for two homologically trivial vector fields with the same invariant volume form. There is also a more general formulation developed by Novikov (see [No]).

Notice that the singularity of $L\left(\Gamma_{p}, \Gamma_{q}\right)$ as $p$ tends to $q$ is of order $\operatorname{dist}^{-2}(p, q)$ and the diagonal $\Delta \subset M \times M$ is of codimension 3; it will contribute with measure zero to the integral. Nevertheless the hypothesis that the vector field $X$ is homologically trivial is necessary.

2.2.1. Example. As in Example 1.2, let $\left.M^{3}=S \widetilde{L(2}, \mathbb{R}\right) / \Gamma$; with the vector fields $X$, $Y$, and $H$ that generate $f_{t}, g_{t}$ the corresponding expanding and contracting horocyclic flows and $h_{t}$ the geodesic flow. The invariant volume form is $v=c \cdot \theta_{X} \wedge \theta_{Y} \wedge \theta_{H}$, where $\theta_{X}, \theta_{Y}$ and $\theta_{H}$ denote the dual 1-forms to the corresponding vector fields, and $c$ is a normalizing constant such that $\int_{M} v=1$. Then, from the relations $[H, X]=2 X$, $[H, Y]=-2 Y$ and $[X, Y]=H$ we obtain the dual relations: $d \theta_{X}=\theta_{X} \wedge \theta_{H}=$ $-\frac{1}{c} i_{Y}(v), d \theta_{Y}=-\theta_{Y} \wedge \theta_{H}=-\frac{1}{c} i_{X}(v), d \theta_{H}=-\frac{1}{2} \theta_{X} \wedge \theta_{Y}=-\frac{1}{2 c} i_{H}(v)$. 
Consequently $v$ is $f_{t}, g_{t}$ and $h_{t}$ invariant as we claimed above. These three vector fields are homologically trivial in the sense of Arnold. Furthermore, we compute their asymptotic Hopf invariants:

$$
\begin{aligned}
& L(X)=\int_{M} c^{2} \theta_{Y} \wedge d \theta_{Y}=0, \\
& L(Y)=\int_{M} c^{2} \theta_{X} \wedge d \theta_{X}=0,
\end{aligned}
$$

and

$$
L(H)=\int_{M}(2 c)^{2} \theta_{H} \wedge d \theta_{H}=-2 c
$$

(where $c^{-1}$ is the volume of $M$ with respect to $\theta_{X} \wedge \theta_{Y} \wedge \theta_{H}$ ).

\section{The Jones-Witten Invariant for Flows}

3.1. In [W] Witten gives a generalization of the Jones Polynomial for links in $S^{3}$ which is valid for a link $L$ in any closed compact 3-manifold $M$. His definition is as follows. Assume $M^{3}$ is a 3-dimensional closed compact manifold and $L \subset M$ is a link (a disjoint union of finitely many non-intersecting circles $S^{1}$ smoothly embedded in $M$ ). Let $P=M \times G$ be a $G$-principal fibre bundle over $M$, where the structure group $G$ might be $U(1)$ or $S U(N)$. Let us denote by $\mathscr{A}$ the space of all connections in $P$ which, since $P$ is topologically trivial, can be identified with the vector space $\Lambda^{1}(M, \mathfrak{g})$ of 1 -forms in $M$ with values in the Lie algebra $\mathfrak{g}$ of $G$; and $\mathscr{G}=\mathscr{C}^{\infty}(M, G)$ be the gauge group of $P$, that is the group of fibre preserving automorphisms of $P$.

Assume $L=\bigcup_{i=1}^{n} C_{i}$ is the disjoint union of smoothly embedded circles $C_{i}$. Then Witten defines:

$$
W_{L}(k)=\int_{\mathscr{L} / \mathscr{G}}\left\{\exp \left(\frac{i k}{4 \pi} \int_{M} \operatorname{tr}\left(A d A+\frac{2}{3} A^{3}\right)\right) \cdot \prod_{C_{i} \subset L} \operatorname{tr}\left(P \exp \int_{C_{\imath}} A\right)\right\} \cdot D A
$$

and he verifies that when $M$ is $S^{3}$, and $G=S U(2)$, this corresponds to values (depending on $k$ ) of the Jones polynomial for the link $L$.

The formlism to justify the meaning of this integral is still not completely done. Ramadas, Singer and Weitsman [RSW] have given a beautiful description based on the arguments of Witten. In it, they use a Heegard splitting of the 3-manifold $M$ as $M=M_{1} \cup_{N} M_{2}$, where $M_{i}$ are 3-manifolds with boundary $\partial M_{i}=N^{2}$, and $N^{2}$ is a compact connected 2-manifold. The glueing is performed by identifying the boundary by an orientation reversing homeomorphism. The contribution to the integral comes from each block of the 3-manifold as a path-integral that corresponds to a holomorphic section of a holomorphic line bundle (the Quillen line bundle) on the space of flat connections over the principal bundle $\left.P\right|_{N} \rightarrow N^{2}$. The whole integral will correspond to the duality pairing between these sections, that is induced by the glueing of $M_{1}$ and $M_{2}$ along $N$ (the orientation reversing map from $N$ to itself is what induces this "dulaity" pairing between the sections). 
If the group $G$ is $U(1)$ or any other abelian group, the understanding of the integral is "easier" as discussed by Witten. When $G$ is $U(1)$ one has:

$$
W_{L}(k)=\int_{\mathscr{b} / \mathscr{G}}\left\{\exp \left(\frac{i k}{4 \pi} \int_{M} A d A\right) \cdot \prod_{C_{i} \subset L}\left(\exp \int_{C_{i}} A\right)\right\} \cdot D A .
$$

To evaluate $W_{L}(k)$, one has to think of $\int_{M} A d A$ as a quadratic form $Q(A)$ on $\mathscr{A}$, $\int_{C_{i}} A$ as a current $T_{c_{i}}(A)$ evaluated on the 1 -forms $A$; and then one has to "complete" the quadratic form, to have a quadratic Gaussian integral over $\mathscr{A}$. The quotient by $\mathscr{G}$ regularizes the term induced by the "determinant" of the quadratic form $Q$, which is

$$
\sqrt{\operatorname{det} d_{A}^{*} d_{A}}
$$

where $d$ is acting on $\operatorname{Ker}\left(d_{A}^{*}\right)$ (the normal direction of the flat connections $\left\{A: F^{A}=\right.$ $0\}$, where $F^{A}$ is the curvature form of the connection A) obtaining the Ray-Singer torsion factor below. Notice that a choice of metric on the manifold was necessary to define the adjoint operators. Then as shown by Witten (following Polyakov, see [P]), after evaluation, one obtains, modulo a phase factor related to the choice of framing:

$$
W_{L}(k)=c(M) \exp \left\{\frac{2 \pi i}{k} \sum_{i, j}\left\langle T_{C_{i}}, G \cdot T_{C_{j}}\right\rangle\right\}=c(M) \exp \left\{\frac{2 \pi i}{k} \sum_{i, j} L\left(C_{i}, C_{j}\right)\right\}
$$

where $G$ is the Green function of the laplacian $\Delta=d^{*} d$ acting on the orthogonal complement of the kernel of $d_{A}=d$ in $\Lambda^{1}(M, \mathfrak{g})$; and $c(M)$ is a constant depending on the Ray-Singer torsion and the choice of framing on the manifold (i.e., a trivialization of its tangent bundle). In the above formula one has to regularize the "self linking number," $L\left(C_{i}, C_{i}\right)$. For this reason Witten defines his invriant for framed and oriented links.

To understand this formula, it is better to explain heuristically how this computation is carried out. If one is computing the quadratic Gaussian integral:

$$
\int_{\mathbb{R}^{n}} e^{i k\langle x, Q x\rangle} e^{\imath\langle b, x\rangle}\left(\pi^{-\frac{n}{2}}\right) d x
$$

on a finite dimensional space, it is necessary to do a change of variables to complete the quadratic form so that one has an integral of the exponential of a quadratic form. One obtains:

$$
\begin{gathered}
e^{\frac{i}{2 k}\left\langle b, Q^{-1} b\right\rangle} \int_{\mathbb{R}^{n}} e^{i k\left\langle x+Q^{-1} b, Q\left(x+Q^{-1} b\right)\right\rangle}\left(\pi^{-\frac{n}{2}}\right) d x \\
=e^{\frac{i}{2 k}\left\langle b, Q^{-1} b\right\rangle}(\operatorname{det} Q)^{-1 / 2}\left(e^{\left(\frac{i \pi}{4} \cdot \operatorname{sign} Q\right)}\right) .
\end{gathered}
$$

In the infinite dimensional case, one considers the completion of the space $\mathscr{C}=\Lambda^{1}(M, \mathfrak{g})$ in the $L^{2}$ norm $\langle$,$\rangle to make it a Hilbert space. For this one needs to$ choose a Riemannian metric on $M$, and the Killing form of the group $G$, but the end result is independent of the choice, modulo a phase factor. Below we also identify . A with its dual by the Riesz representation theorem. 
With this interpretation, we need to observe that the quadratic form $\int_{M} A \wedge d A$ needs to be symmetrized. For this we use the Hodge decomposition of the space of 1-forms:

$$
\Lambda^{1}(M, \mathfrak{g})=\mathscr{H} \oplus d\left(\Lambda^{0}\right) \oplus d^{*}\left(\Lambda^{2}\right),
$$

into mutually orthogonal direct summands. Here $\mathscr{H}$ is the subspace of harmonic forms, $\Lambda^{i}$ the vector space of $i$-forms on $M$ with values in the Lie algebra; and $d_{A}=d$ is acting on the orthogonal complement of its kernel, that is, on $d^{*}\left(\Lambda^{2}\right)$. To make it act from the Hilbert space into itself, we compose $d$ with its adjoint $d^{*}$, obtaining the Laplacian, which is symmetric. On the other hand, the action on $\mathscr{A}$ by the gauge group $\mathscr{G}$ is being taken care by dividing by the "volume" of $\mathscr{G}$, since the infinitesimal action of $\mathscr{G}$ on $T_{A}(\mathscr{C})$, the tangent space at $A$ of . $\mathscr{C}$ is by the operator $d_{A} ;$ then

$$
\operatorname{vol}(\mathscr{G})=\sqrt{\operatorname{det} d_{A}^{*} d_{A}}
$$

Integration of the connection form $A$ with respect to the knot $C_{\imath}$ is thought of as an element on the dual of the Hilbert space, or as a current acting on the space of 1 -forms, and it is denoted by $T_{C_{\imath}}$. We have then,

$$
\begin{aligned}
W_{L}(k)= & \int_{\mathscr{C} / \mathscr{G}}\left\{\exp \left(\frac{i k}{4 \pi} \int_{M} A d A\right) \cdot \prod_{C_{i} \subset L}\left(\exp \int_{C_{i}} A\right)\right\} \cdot D A \\
= & \frac{1}{\operatorname{vol} \mathscr{G}} \int_{\mathscr{C}}\left\{\exp \left(\frac{i k}{4 \pi} \int_{M} A d A\right) \cdot \exp \left(\sum_{i} \int_{C_{\imath}} A\right)\right\} \cdot D A \\
= & \operatorname{vol}(\mathscr{G})^{-1} e^{\frac{2 \pi \imath}{k} \sum_{i, j}\left\langle T_{C_{\imath}}, G \cdot T_{C_{\jmath}}\right\rangle} \\
& \times \int_{\mathscr{C}}\left\{e^{\frac{\imath k}{4 \pi}\left\langle A+\sum G \cdot T_{C_{\imath}}, \Delta\left(A+\sum G \cdot T_{C_{j}}\right)\right\rangle}\right\} \cdot D A \\
= & c(M) \exp \left\{\frac{2 \pi i}{k} \sum_{i, j}\left\langle T_{C_{i}}, G \cdot T_{C_{\jmath}}\right\rangle\right\},
\end{aligned}
$$

where the constant $c(M)$ is the Ray-Singer torsion times the phase factor depending on the framing of the manifold, as treated in Atiyah [At] Sect. 7.2.

3.2. The Definition for Flows (Abelian Case). In the case of a measure preserving flow determined by a vector field $X$ (where $\mu$ denotes the measure in $\mathscr{B}(X)$ ) we propose the following extension of the Jones-Witten functional.

We will start studying the holonomy and the asymptotic holonomy around the orbits of the flow.

Notice that we can then consider for any Lie group $G$ :

$$
\operatorname{Hol}_{\tilde{\Gamma}_{t, p}}(A)=P \exp \int_{\Gamma_{t, p}} A,
$$

the path ordered exponential integral of the connection form (see Nelson [N, pp. 15-16]). Hence by the homological properties of the asymptotic cycles (they are 
1-cycles in homology), in the abelian case we define the asymptotic holonomy as:

$$
\operatorname{Hol}_{\Gamma_{p}}(A)=\lim _{t \rightarrow \infty} \exp \int_{\tilde{\Gamma}_{t, p}}\left(\frac{1}{t} A\right)=\exp \int_{\Gamma_{p}} A,
$$

where the last integral is defined by the limiting procedure and $p$ is a quasi-regular point in $M$ as defined in Sect. 1. Hence it is natural to consider the average holonomy for the vector field. The Ergodic Theorem implies that the limit is well defined for a quasi-regular point. In the abelian case we do not need to take the path ordered exponential but just the standard exponential. The nonabelian case is more complicated and will be treated in Sect. 4 below.

Definition. We will assume that the structure group is $U(1)$. If $X$ is a vector field, $\mu$ is a measure in $\mathscr{B}(X)$ invariant under translations by the flow generated by the vector field, and we have the inducd asymptotic cycles as before, we define the average holonomy of a U(1)-connection A over all the asymptotic cycles as:

$$
\operatorname{Hol}_{X, \mu}(A)=\exp \int_{M} A(X) d \mu .
$$

One might interpret asymptotic holonomy as the asymptotic value of the Berry phase or Bohm-Aharonov effect.

The idea to define the average holonomy is inspired by the interpretation of the asymptotic cycles by means of winding cycles, namely, we would like to be able to write a multiplicative average of all the holonomies $\operatorname{Hol}_{\Gamma_{p}}(A)$, with respect to $p \in M$ and to the $X$-invariant measure $\mu$. In the abelian case (gauge group $U(1)$ ) this can be done because

$$
\lim _{t \rightarrow \infty} \exp \int_{M}\left(\int_{\tilde{\Gamma}_{t, p}}\left(\frac{1}{t} A\right)\right) d \mu(p)
$$

is equal, in term of winding cycles, to

$$
\exp \int_{\Gamma} A=\exp \int_{M} A(X) d \mu
$$

where $\Gamma$ corresponds to the "averaged" $\Gamma_{p}$ on $H_{1}(M, \mathbb{R})$ with respect to the measure $\mu$, i.e. in terms of winding cycles we have $\int_{\Gamma} A=\Psi_{\mu}(A)$. This argument also implies that the trace of the average holonomy is gauge invariant in the abelian case, since parallel translation changes by conjugation at every finite time, it can also be seen as follows. If $A$ changes by a gauge transformation into $A+d \alpha$ then the integral:

$$
\int_{M}(A(X)+d \alpha(X)) d \mu=\int_{M} A(X) d \mu,
$$

because

$$
\int_{M} d \alpha(X) d \mu=0
$$

since the measure is $f_{t}$-invariant. 
Definition. Let $M$ be a closed 3-manifold, $X$ a vector field, $\mu$ an invariant probability measure and $\pi: P \rightarrow M$ a topologically trivial principal fibre bundle over $M$ with structure group $G=U(1)$. Let $\mathscr{b}$ be the space of all $G$-connections and $\mathscr{G}$ the gauge group. Then we define the Jones-Witten functional for the flow generated by $X$ with respect to the measure $\mu$ as follows:

$$
W_{X, \mu}(k)=\int_{\mathscr{C} / \mathscr{G}}\left\{\exp \left(\frac{i k}{4 \pi} \int_{M} \operatorname{tr}(A d A)\right) \cdot\left(\operatorname{Hol}_{X, \mu}(A)\right)\right\} \cdot D A
$$

where the asymptotic holonomy is defined as above.

Remark. In the abelian case if the $X$-invariant probability measure $\mu$ is defined by the sum of a finite number of invariant measures $\mu_{j}, j=1, \ldots, n$; each invariant by the action of the flow generated by the vector field $X$, and each one uniformly distributed on a periodic orbit of the flow; then our definition agrees with the definition of Witten for a link (thinking of the measures as Dirac measure concentrated on the connected components of the link). This is so because in this case the averaging procedure for the holonomy is a finite multiplicative average with respect to the periodic orbits of the flow on which the measure is supported.

It now becomes easy (after the computations described in Sect. 3.1, and from the results of Arnold described in Sect. 2.2), to do explicit computations when the structure group is $U(1)$.

We think of

$$
\int_{\Gamma_{p}} A:=\lim _{t \rightarrow \infty} \int_{\tilde{\Gamma}_{t, p}}\left(\frac{1}{t} A\right)
$$

as a current

$$
A \mapsto T_{\Gamma_{p}}(A)
$$

evaluated on the elements of $\mathscr{A}=\Lambda^{1}(M, \mathfrak{u}(1))$, the connection forms. The average can be thought of also as a current:

$$
T_{\mu}(A)=\int_{M} A(X) d \mu
$$

and we have that the average of the currents $T_{\Gamma_{p}}$ for $p \in M$ with respect to $\mu$ is precisely $T_{\mu}$ by the interpretation above of the average by means of winding cycles. One obtains therefore the following:

\section{Lemma.}

$$
W_{X, \mu}(k)=c(M) \exp \left\{\frac{2 \pi i}{k} \int_{M \times M} L\left(\Gamma_{p}, \Gamma_{q}\right) d \mu(p) d \mu(q)\right\},
$$

where $L\left(\Gamma_{p}, \Gamma_{q}\right)$ is the asymptotic linking number defined by Arnold (the asymptotic Hopf invariant).

Proof. In the description of the formula for the case of a link in Sect. 3.1, we were considering the current $\sum_{\imath} T_{C_{\imath}}$. In our case we have $\int_{M} T_{\Gamma_{p}} d v(p)$, so that in the formula 
for completing the quadratic form, one obtains:

$$
c(M) \exp \left\{\frac{2 \pi i}{k} \int_{M \times M}\left\langle T_{\Gamma_{p}}, G \cdot T_{\Gamma_{q}}\right\rangle d v(p) d v(q)\right\},
$$

which is exactly the exponential of Arnold's generalized Hopf invariant.

Remark. It is interesting to notice that in the case when the measure is smooth, we do not have to choose a framing to regularize the self-linking numbers of the asymptotic cycles, as we pointed out above, because the singularity at the diagonal of $M \times M$ is of order $O\left(r^{-2}\right)$ while the codimension of the diagonal is 3. Furthermore, requiring the vector field to be homologically trivial corresponds to choosing a "good normal framing" for the asymptotic cycle. Also by taking the average one takes care of the phase scaling. However, for general measures, one might have singularities leading to the necessity of the choice of framing as is the case for links.

This implies our main result for the abelian case, namely that the generalized Jones-Witten formula is computable for flows associated to vector fields:

Theorem. Let $M$ be a closed 3-manifold, $\pi: P \rightarrow M$ a topologically trivial U(1)principal bundle, $X$ a vector field on $M$ and $v$ a normalized volume form. Assume that the measure defined by $v$ is invariant under the action of the flow generated by the vector field $X$. Assume furthermore that $X$ is homologically trivial with respect to $v$. That is, there exists a 1 -form $\alpha$ such that $d \alpha=i_{X}(v)$. Then

$$
W_{X, v}(k)=c(M) \exp \left(\frac{2 \pi i}{k} \int_{M} \alpha \wedge d \alpha\right) .
$$

Where $c(M)$ is the Ray-Singer torsion times a phase factor, as described above.

3.3 Example. Let $M=S \widetilde{L(2}, \mathbb{R}) / \Gamma$, where $\Gamma$ is a discrete subgroup as in Examples 1.2 and 2.2.1. Let $X, Y$ be the vector fields generating the expanding and contracting horocyclic flows respectively; and $H$ be the vector field generating the geodesic flow. We then have the relations $[H, X]=2 X,[H, Y]=-2 Y$ and $[X, Y]=H$.

Their dual 1-forms: $\theta_{X}, \theta_{Y}$ and $\theta_{H}$ satisfy the relations: $d \theta_{X}=\theta_{X} \wedge \theta_{H}=$ $-\frac{1}{c} i_{Y}(v), d \theta_{Y}=-\theta_{Y} \wedge \theta_{H}=-\frac{1}{c} i_{X}(v), d \theta_{H}=-\frac{1}{2} \theta_{X} \wedge \theta_{Y}=-\frac{1}{2 c} i_{H}(v)$.

Recall that the constant $c$ is defined such that the normalized form $v=c \theta_{X} \wedge \theta_{Y} \wedge$ $\theta_{H}$ is the probability measure for $M$, i.e. $\int_{M} v=1$.

Since the vector fields $X, Y$ and $H$ are homologically trivial, they satisfy Arnold's conditions as computed in Sect. 2.2.1. We verified that $L(X)=0, L(Y)=0$ and $L(H)=-2 c$ are their asymptotic Hopf invariants respectively. It then follows that their corresponding generalized Jones-Witten functionals can be effectively computed.

This proves:

Theorem. For $M=S \widetilde{L(2,}, \mathbb{R}) / \Gamma$, with vector fields $X, Y$, and $H$ as before; and $v$ the normalized volume form with normalizing constant $c$, we have that the values of the corresponding Jones-Witten functional are:

$$
W_{X, v}(k)=W_{Y, v}(k)=c(M)
$$

and

$$
W_{H, v}(k)=c(M) \exp \left(-\frac{4 \pi i c}{k}\right)
$$


Notice that if $N^{2}$ is an orientable compact surface of genus $g \geq 2$, then the unit

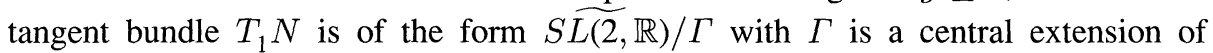
$\pi_{1}(N)$, the fundamental group of $N$, hence there are many examples of 3-manifolds with invariants as above and the normalizing constant is $c=\frac{1}{4 \pi^{2}(g-1)}$.

Another set of examples of the same kind can be obtained by considering the Brieskorn manifold $M(p, q, r)$ defined as the intersection of the complex singular hypersurface given by $H=\left\{\left(z_{1}, z_{2}, z_{3}\right) \in \mathbb{C}^{3} \mid z_{1}^{p}+z_{2}^{q}+z_{3}^{r}=0, p, q\right.$, and $r$ coprime, and $\left.\frac{1}{p}+\frac{1}{q}+\frac{1}{r}<1\right\}$ with the unit sphere $s^{5}=\left\{\left|z_{1}\right|^{2}+\left|z_{2}\right|^{2}+\left|z_{3}\right|^{2}=1\right\}$.

Milnor [M] and Dolgachev [D] have shown that $M(p, q, r)$ is diffeomorphic to a quotient $S \widetilde{L(2}, \mathbb{R}) / \Gamma$, where $\Gamma$ is the commutator of a central extension of the Schwartz triangle group $\Sigma(p, q, r)$. For all these manifolds our invariant is computable and has the values as stated in the last theorem. Under the above hypothesis Brieskorn has shown that $M(p, q, r)$ are homology 3-spheres and therefore every flow is automatically homologous to zero by Poincaré duality.

\section{The Non-Abelian Case}

If the gauge group $G$ is a connected simply connected non abelian Lie group, for instance $S U(N)$, the definition of the average holonomy has to be dealt more carefully. The purpose of this section is to give a rigorous meaning and proof of the existence of the average asymptotic Wilson line.

As before, let $X$ be the vector field with associated flow $f_{t}$ and invariant measure $\mu$ on $M$. Let $A$ be a connection form on the trivial principal $G$-bundle over $M$. In order to define the asymptotic Wilson line through an orbit we need the following concepts. As in Sect. 1, we will denote the oriented segment of the orbit of the flow $f_{t}$ from the point $p$ to the point $f_{t}(p)$ by $\left[p, f_{t}(p)\right]$, and the cycle formed after joining its end points by $\tilde{\Gamma}_{t, p}=\left[p, f_{t}(p)\right] \cup \gamma_{f_{t}(p), p}$. As in Arnold [A], the closed curves $\left\{\gamma_{p, q}\right\}$ can be chosen to depend piecewise smoothly on $p$ and $q$ for almost all $p$ and $q$ in $M$. We will denote the holonomy determined by the connection $A$ on the closed curve $\tilde{\Gamma}_{t, p}$ by $H_{A}(t, p)$ and by $P_{A}(t, p)$ the parallel transport along the orbit $\left[p, f_{t}(p)\right]$ of the flow and think of them as maps:

$$
P_{A}, H_{A}: \mathbb{R} \times M \rightarrow G .
$$

We observe that $H_{A}$ is a measurable function and $P_{A}$ is a continuous cocycle with values in $G$ i.e.:

$$
P_{A}\left(t_{1}+t_{2}, p\right)=P_{A}\left(t_{2}, f_{t_{1}}(p)\right) \circ P_{A}\left(t_{1}, p\right) .
$$

Furthermore, if we change the connection $A$ to a connection $A^{g}$ by the gauge transformation $g$ then $P_{A}$ is cohomologous to $P_{A^{g}}$. This means that there exists a measurable function $C: M \rightarrow G$ such that $F_{A^{g}}(t, p)=\left\{C\left(f_{t}(p)\right)\right\}^{-1} F(t, p) C(p)$ (see [CFS]). Instead of dividing the connection by $t$, as in the abelian case, in order to take the average limiting behaviour of both holonomy or parallel transport we have to take now the " $t$-root" of both elements of the Lie group. We do this as follows: We fix once and for all a representation of the Lie group in $\mathbb{C}^{n}$ and think of its elements as $n \times n$ complex matrices via this representation. The function that assigns to each matrix its set of eigenvalues is a continuous function from $G$ into $\operatorname{Sym}^{n}\left(\mathbb{C}^{*}\right)$, the 
$n$-fold symmetric product of the multiplicative group of nonzero complex numbers. Since $P_{A}$ is continuous and homotopic to a constant it follows that we can take a continuous branch of the logarithms of its eigenvalues. Thus we obtain the map:

$$
\mathscr{P}_{A}: \mathbb{R} \times M \rightarrow \operatorname{Sym}^{n}(\mathbb{C})
$$

described by

$$
\mathscr{P}_{A}(t, p)=\left\{\frac{1}{t} \log \lambda_{1}(t, p, A), \ldots, \frac{1}{t} \log \lambda_{n}(t, p, A)\right\} \in \operatorname{Sym}^{n}(\mathbb{C}),
$$

where $\lambda_{\imath}(t, p, A)$ denote the eigenvalues of $P_{A}(t, p)$. Since the curves $\left\{\gamma_{p, q}\right\}$ depend measurably on $p$ and $q$ and their length is uniformly bounded one can choose branches of the logarithms of the eigenvalues of $H_{A}(t, p)$ to obtain a function:

$$
\mathscr{H}_{A}(t, p)=\left\{\frac{1}{t} \log \mu_{1}(t, p, A), \ldots, \frac{1}{t} \log \mu_{n}(t, p, A)\right\} \in \operatorname{Sym}^{n}(\mathbb{C}),
$$

such that

$$
d\left(\mathscr{P}_{A}(t, p), \mathscr{H}_{A}(t, p)\right) \leq \frac{b(t, p)}{t},
$$

where $b$ is a bounded measurable function, $\mu_{i}(t, p, A)$ denote the eigenvalues of $H_{A}(t, p)$ and $d$ denotes a complete distance in $\operatorname{Sym}^{n}(\mathbb{C})$.

Lemma 1. The limit $\lim _{t \rightarrow \infty} \mathscr{P}_{A}(t, p)$ exists for $\mu$-almost every point of $M$ and therefore by (*) also $\lim _{t \rightarrow \infty} \mathscr{H}_{A}(t, p)$ exists and both limits are equal. Furthermore, this limit is independent of gauge transformations and the original choice of the branch of the logarithm.

Proof. As we remarked, $P_{A}$ is a cocycle with values in $G$. Now we proceed as in Oseledec's proof of the Multiplicative Ergodic Theorem (see [O, pp. 228-230]). Our next aim is to prove that there exists an extension of the flow such that in this extension the cocycle $P_{A}$ is Lyapunov homologous to a cocycle with values in a compact abelian group which in our case is a maximal torus, $\mathfrak{T}$ of $G$. One can assume that under the given representation of $G$ the subgroup $\mathfrak{T}$ correspond to diagonal matrices. Let $N=G / \mathfrak{T}$ be the quotient of $G$ by $\mathfrak{T}$. For $g \in G$ let $[g] \in N$ denote the left coset $g \mathfrak{T}$ corresponding to $g$. Define the flow $\phi_{t}: M \times N \rightarrow M \times N$ by: $\phi_{t}(p,[g])=\left(f_{t}(p),[F(t, p) g]\right)$. The fact that $F(t, p)$ is a cocycle implies that $\phi_{t}$ is indeed a flow i.e.: $\phi_{t+s}=\phi_{t} \circ \phi_{s}$. The flow $\phi_{t}$ preserves the measure $\tilde{\mu}$ which is the direct product of the measure $\mu$ in $M$ and the measure induced by Haar measure in $N$. Then the flow $\phi_{t}$ is an extension of the flow $f_{t}$. Let $T: N \rightarrow G$ be a measurable section of the bundle $\pi: G \rightarrow G / \mathfrak{T}$ (of course a continuous section might not exist but it is always possible to find a measurable section). With this section one can find a measurable trivialization of the bundle.

Therefore one can think of the function $\mathfrak{p}: \mathbb{R} \times M \rightarrow \operatorname{Sym}^{n}(\mathbb{C})$ given by

$$
\mathfrak{p}(t, p)=\left\{\log \lambda_{1}(t, p), \ldots, \log \lambda_{n}(t, p)\right\} \in \operatorname{Sym}^{n}(\mathbb{C})
$$

as a "cocycle" with values in the quotient of $\mathbb{C}^{n}$ by the action of the symmetric group and defined in an extension of our original flow. Therefore, by the Ergodic Theorem for abelian cocycles, i.e. the Multiplicative Ergodic Theorem, which in the case of abelian cocycles reduces to Birkhoff's Ergodic Theorem (see [O, p. 217]) we have that:

$$
\lim _{t \rightarrow \infty} \frac{1}{t} \mathfrak{p}(t, p)=\lim _{t \rightarrow \infty} F(t, p)
$$


exists for almost every point and hence, both limits in the lemma exist for almost every point as in the case of Oseledec's Theorem. Since when $\lim _{t \rightarrow \infty} \mathscr{H}_{A}(t, p)$ exists is independent of gauge transformations, because it is the holonomy of the connection along closed curves, we have that the limits are invariant under the gauge group. This also follows from the fact that $P_{A}$ is cohomologous to $P_{A g}$. Also the limits are independent of the original choice of the continuous branch of the logarithms because any other choice differs by a constant function equal to an integer multiple of $2 \pi i$.

Now define the function

$$
w: M \times \mathscr{b} \rightarrow \mathbb{C}
$$

by,

$$
w_{A}(p):=w(p, A)=\sum_{i=1}^{n} e^{\lim _{t \rightarrow \infty} \frac{1}{t} \lambda_{i}(t, p)} .
$$

Definition. The number $w_{A}(p)$ is the asymptotic Wilson line of the orbit through $p$, when defined. It exists for almost all $p \in M$ with respect to the measure $\mu$.

We notice that:

1. $w_{A}(p)$ is a real number when $G=S U(2)$.

2. The definition of asymptotic Wilson line is the same as the usual trace of the holonomy in case the orbit is periodic, and it is invariant by the gauge group for any orbit where it exists. It agrees with the abelian case, since in this case taking the $t$-root of the element of the group obtained by parallel transport along a curve gives the same result as the one obtained by integration of the connection form divided by $t$ along the curve.

3. By the Ergodic Theorem and the cocycle condition, the function $w_{A}(p)$ is constant along the orbit of $p$ by the flow.

4. The Ergodic Theorem implies then that when the flow is ergodic the function $w_{A}(p)$ is $\mu$-almost everywhere constant. Hence if the flow is uniquely ergodic $w_{A}(p)$ depends only on the flow and the connection form.

From the above we have the following:

Theorem. The average asymptotic Wilson line of the connection with respect to the measure $\mu$ defined by:

$$
w_{\mu}(A)= \begin{cases}0, & \text { if } \mu\left(w_{A}^{-1}(0)\right)>0 \\ \exp \int_{M} \log w_{A}(p) d \mu(p), & \text { otherwise }\end{cases}
$$

is well defined and is gauge invariant. In this formula one chooses any measurable branch of the logarithm.

In this case we are taking the multiplicative average of the Wilson lines with respect to the measure. Then if the measure is uniformly distributed along a finite number of closed orbits it corresponds exactly to the product of the non-vanishing Wilson lines. In the abelian case this corresponds exactly to the average asymptotic holonomy. We observe that in this definition it is necessary to integrate the flow, unlike the abelian case where the definition depends only on the vector field and the connection.

Thus one is able to generalize Witten's invariant for flows. 
Theorem. The following formula generalizes the Jones-Witten invariant for a vector field with an invariant measure:

$$
W_{X, \mu}(k)=\int_{\mathscr{C} / \mathscr{G}} \exp \left(\frac{i k}{4 \pi} \int_{M} \operatorname{tr}\left(A d A+\frac{2}{3} A^{3}\right)\right) w_{\mu}(A) D A .
$$

In the abelian case this corresponds to the definitions of Sect. 3 above. And in the case of mesures supported on a finite number of closed orbits and uniformly distributed along them, it is essentially Witten's formula for links on the 3-manifold.

Acknowledgements. The authors would like to thank Prof. Jim Eells for his support and encouragement. They would also like to thank Prof. Abdus Salam, the International Atomic Energy Agency and UNESCO for hospitality at the International Center for Theoretical Physics, Trieste.

\section{References}

[A] Arnold, V.I.: The asymptotic Hopf invariant and its applications. Materialy Vsesoyuznoi Shkoly po Differentsialnym Uravneniyam s Beskonechnym Chislom Nezavisimyh i po Dynamicheskim Sistemam c Beskonechnym... (in Russian), 1973-74; English transl. in Sel. Math. Sov. 5 (4), 327-345 (1986)

[At] Atiyah, M.: The geometry and physics of knots. Cambridge: Cambridge University Press 1990

[AB] Atiyah, M., Bott, R.: The Yang Mills equations over Riemann surfaces. Phil. Trans. R. Soc. Lond. A 308, 523-615 (1982)

[CFS] Cornfeld, I.P., Fomin, S.V., Sinai, Y.G.: Ergodic theory, Berlin, Heidelberg, New York: Springer 1982

[D] Dolgacev, I.V.: Conic quotient singularities of complex surfaces. Funct. Anal. Appl. 8(2), 160-161 (1974)

[KC] Khesin, B.A., Chekanov, Yu.V.: Invariants of the Euler equations for ideal or barotropic hydrodynamics and superconductivity in D dimensions. Physica D 40, 119-131 (1989)

[KN] Kobayashi, S., Nomizu, K.: Foundations of differential geometry, Vol. 1. New York: Wiley 1963

[M] Milnor, J.: On the 3-dimensional Brieskorn manifolds $M(p, q, r)$. In: Knots, groups and 3manifolds (Neuwirth, ed.) Ann. Math. Studies. Princeton, NJ: Princeton Univ. Press 1975, pp. 175-225

[N] Nelson, E.: Topics in dynamics. I. Flows. Princeton: Princeton Univ. Press 1969

[No] Novikov, S.P.: The analytic generalized Hopf invariant. Many-valued functionals (in Russian). Uspekhi Mat. Nauk (1984); English transl. in Russ. Math. Surv. 39 (5), 113-124 (1984)

[O] Oseledec, V.I.: A multiplicative ergodic theorem. Lyapunov characteristic numbers for dynamical systems. Trans. Moscow Math. Soc. 19, 197-231 (1968)

[P] Polyakov, A.M.: Fermi-Bose transmutations induced by gauge fields. Mod. Phys. Lett. A 3, 325 (1988)

[RSW] Ramadas, T.R., Singer, I.M., Weitsman, J.: Some comments on Chern-Simons gauge theory. Commun. Math. Phys. 126, 409-420 (1989)

[Sc] Schwartzman, S.: Asymptotic cycles. Ann. Math. 66 (2), 270-284 (1957)

[Su] Sullivan, D.: Cycles for the dynamical study of foliated manifolds and complex manifolds. Invent. Math. 36, 225-255 (1976)

[T] Tabachnikov, S.L.: Two remarks on the asymptotic Hopf invariant (in Russian). Funktsional'nyi Analiz i Ego Prilozheniya (1990); English transl. in Funct. Anal. and its Appl. 24(1) (1990)

[W] Witten, E.: Quantum field theory and the Jones polynomial. Commun. Math. Phys. 121, 351-399 (1989) 